\title{
Integrated sensors and microsystems for biological and environmental applications
}

\author{
F. J. Ramirez-Fernandez ${ }^{*, 1}$, W. J. Salcedo ${ }^{1}$, E. Galeazzo ${ }^{1}$, H. E. M. Peres ${ }^{1}$, P.Hidalgo $^{1}$, \\ N. Peixoto ${ }^{2}$, and J. C. Rubim ${ }^{3}$ \\ ${ }^{1}$ Laboratório de Microeletrônica, Departamento de Engenharia de Sistemas Eletrônicos, Escola \\ Politécnica da Universidade de São Paulo, Av. Prof. Luciano Gualberto, trav. 3, 158, 05508-900 \\ São Paulo, Brazil \\ 2 Krasnow Institute, George Mason University, USA \\ ${ }^{3}$ Instituto de Química da Universidade de Brasília, C.P. 04478, 70919-970 Brasilia, DF, Brazil
}

Received 25 March 2004, revised 7 June 2004, accepted 9 June 2004

Published online 1 September 2004

PACS 61.43.Gt, 73.61.Jc, 73.61.Ey

This work presents a brief review and some significant results about integrated sensors andmicrosystems such as microelectrode array (MEA) for biological cell culture studies and gas sensors based on nanostructured materials as porous silicon (PS) and tin oxide $\left(\mathrm{SnO}_{2}\right)$. Developed MEA provides electrical access to biological neurons culture for signal recording and stimulation showing that the charge injection limit can be lowered to less than $100 \mathrm{nC} / \mathrm{cm}^{2}$. The gas sensors based on PS material are investigated by two approaches: electrical and optical material properties change as function of gas species and concentrations. A model is proposed for PS electrical impedance behavior and the electron transfer mechanism is identified as responsible for the photoluminescence (PL) quenching characterized during essays. Gas sensors based on $\mathrm{SnO}_{2}$ doped with nickel are studied and a good sensitivity for $\mathrm{SO}_{2}$ into environment is verified.

(C) 2004 WILEY-VCH Verlag GmbH \& Co. KGaA, Weinheim

\section{Introduction}

The maturity reached on microelectronics technology for fabrication of integrated circuits has been applied as the basis for the development of miniaturized systems that include electronic devices, sensors and actuators integrated on a chip. Microelectromechanical systems (MEMS) have been developed for applications in many areas beyond electronics such as mechanics, analytical chemistry, biochemistry, thermodynamics and environment monitoring. Attractive characteristics offered by microelectronics are miniaturization, device integration, batch fabrication, reliability, reduced cost, low response time, signal processing and automatization. Results obtained with this technology show new horizons in the industrial scenery for the development of innovative applications. The paradigm in that emergent industrial reality needs to be inserted in an ambivalent context where more appropriate strategic could opt for preferential investment in sensor technology or in systems associated to data processing [1].

In sensor technology, special importance has been given to sensors materials as conductive ceramic, different kinds of polymers and porous silicon (PS), among other advanced materials. The largest attractiveness is provided by compatibility of available conventional processes in Microelectronics to fabricate MEMS with those materials. In that context and with base on the excellent results obtained with the development of PS technology, microelectrode arrays (MEA) and electronic nose, the Integrated Sensors and Microsystem group has been incorporating the development of smart sensors with special attention

\footnotetext{
"Corresponding author: e-mail: jramirez@Ime.usp.br
} 
to expert systems applied to patterns recognition using integrated sensors in several fields of applications including, among others, microbiology and environmental control.

\section{Neuroelectronic matrices}

Microelectrode arrays (MEAs) have been used for the last three decades in cellular biology field as tools for probing electrophysiologic activity either in vitro or in vivo. The availability and development of microelectromechanical systems (MEMS) techniques has enabled the fabrication of three dimensional structures which add spatial resolution and cell development control [2]. Moreover, the miniaturization of sensors that can be implemented along with the electrodes, such as temperature and $\mathrm{pH}$ sensors, enables the production of microsystems which will eventually bypass biomedical equipment and provide enough data as to define neuronal activity and system status.

Although a high control of neuronal systems be desirable, a high connectivity and, therefore, complexity of neuronal networks makes very difficulty to analyse the amount of data available from such systems. Simplification of neuronal networks that can be isolated, maintained or even formed in vitro with a limited quantity of neurons has proven useful for understanding transfer functions and describing basic characteristics of circuits such as pattern generators or rhythmic activity [3]. Such neuronal networks can also be used as sensors for environmental conditions or drug influence on the whole organism. The first step towards such systems is the analysis of cellular adhesion and growth over micromachined substrates, which can then be further developed with onboard circuitry and electrodes. We present here results on the electrical stimulation of Helix aspersa neurons in culture on microelectrode arrays.

\subsection{Microelectrode array fabrication}

Planar microelectrode arrays containing 100 electrodes were fabricated as previously reported [4]. Briefly, metal tracks are photolithographically defined on a glass substrate; passivation is either a duplex layer of ECR silicon nitride followed by PECVD thick oxide or polyimide (PI 2525, HD Microsystems). Individual electrodeposition of platinum black on electrodes is performed in order to obtain ohmic impedance below $50 \mathrm{k} \Omega$ (at $1 \mathrm{kHz}$, sinusoidal excitation wave). Alternatively electrodes are coated with a conductive polymer (polyaniline or polypyrrole) in order to ensure stability during experimental conditions [4]. Medium and long-term (from 10 hours to 10 days) characterization is performed in order to probe electrode stability. The electrode-electrolyte interface has not been modified either by means of mechanical movement or variation of the chemical composition of the electrolyte.

\subsection{Signal recording and stimulation}

Computer controlled acquisition and stimulation is performed by means of virtual instrumentation developed with LabVIEW ${ }^{\circledR}$. Signal acquisition can be performed at 32 channels differentially and input levels are set accordingly to signal variation observed during the experiment. Upon identification of active electrodes a maximum of four sites are chosen and saved for subsequent analysis. Cells placed over the electrodes can be visually identified and respectively accessed by means of differentially recording from neighbouring electrodes. Stimulation threshold for isolated cells is then probed by applying charge balanced square pulses in trains between electrodes, which are disposed across the cell body. Threshold is defined as the electrical stimulus that repeatedly elicits extracellular action potentials to each of the applied pulses. The current profile depends on the electrode impedance and seal to the cell. Charge injection threshold is found to be $0.08 \mu \mathrm{C} / \mathrm{cm}^{2}$. Depending on time in culture, threshold can vary by one order of magnitude. Nevertheless, this value is much lower than the threshold usually found for neural implants, such as the retinal [5] due to the controlled environment. Then, the determination of lower baseline for neural prosthesis can start by looking at some cultured cells thresholds. We were also able to inhibit neuronal activity by means of electrical stimulation (unidentified neurons from the parietal ganglion). In this case the spontaneous activity is in the range of 4 to 12 spikes/s. As soon as the stimulus 
sequence begins the activity is completely depressed, showing the inhibitory effect observed in roughly $20 \%$ of the unidentified neurons maintained in culture for more than 10 days.

\section{Gas sensors based on nanomaterials}

Nowadays there is an increasing interest on the monitoring and control of environment conditions like the air pollution levels and hazardous gases. Thus, there is also an increasing demand for environment monitoring by mean of selective, fast, compact, ease to operate and low cost systems. In this context, materials nanofabricated for sensing application that are compatible with integrated circuits tecnology can be a solution. Nanomaterials like PS and tin oxide $\left(\mathrm{SnO}_{2}\right)$ have attractive characteristics such as high specific area and high chemical surface activity. Nevertheless these materials show elevated sensitivity, properties as selectivity and stability need to be improved and they are important subject of investigation in the present, as discussed forward.

\subsection{Porous silicon gas sensors}

PS layers are obtained conventionally by anodic etching of crystalline silicon into fluoridric acid solution. Morphologically, this material has a structure similar to a quantum sponge, with porous and crystallites with dimensions varying from units of nanometers to tens of micrometers and it is classified in function of pores sizes as: microporous (with a pore size greater than $50 \mathrm{~nm}$ ), mesoporous (pore size of 5-50 $\mathrm{nm}$ ) or nanoporous (pore size less than $5 \mathrm{~nm}$ ). In the past decade significant development has been made towards the understanding and applicability of PS material. Interesting characteristics as photoluminescence (PL) at room temperature and topics about electronic and optical properties have been researched and discussed in the scientific community [6].

PS is also a very interesting material in the field of gas sensors due to its high surface to volume ratio (typically about $500 \mathrm{~m}^{2} / \mathrm{cm}^{3}$ ) and high reactivity to the environment. Other advantages of this material are low cost, room temperature operation and possible integration with electronic circuits.

With the adsorption of molecules of different gas species on PS surface many properties, such as PL and electrical conductivity, will change [7] in such a way that these parameters may be monitored and related to physical reactions, environmental conditions, or even to air quality, which makes PS a promising material for a wide range of applications ranging from environmental gas sensing to chemical detection in very small concentrations [8]. As examples, analysis of humidity and organic molecules as acetone and ethanol [9] as well as low concentrations of $\mathrm{NO}_{2}[10]$ are reported.

Despite the good PS sensitivity to humidity and organic vapors, problems such as sensor stability, reversibility and selectivity must be investigated, towards to obtain commercially useful devices. We believe most of problems related with reversibility are solvable by tuning silicon anodization conditions, and proposing alternatives to passivate PS layers. Many efforts have also devoted to introduce others materials in the porous network, forming different nanocomposites, with the objective to form structures with particular sensing properties [11].

In the following we will report some of ours recent results of PS in the sensors field, showing its advantanges and drawbacks.

\subsubsection{Local conductivity effect on PS gas sensors}

As electrical conductance of PS devices is strongly dependent of on the environmental conditions to which they are submitted, it can be exploited to distinguish substances (or molecules) parametrically. In such way, it is necessary to clarify the conduction mechanisms responsible for electrical properties of PS. Electrical transport in silicon crystallites, Schottky rectification, Poole Frenkel conduction are some of theories suggested to explain the behavior of PS devices electrical transport from dc measurements [8]. Through ac measurement it is possible to analyze local conductivity of this material and its frequency dependence [12]. 
In our group prototypes of gas sensors based on oxidized PS layer were developed and they have been exposed to two different substances with polar molecules (acetone and ethyl alcohol) along with control environmental conditions (vacuum) [9]. Al/PS/Si/Al devices with different perimeter/area relation could be repeatedly used and tested in different atmospheres, thus proving that the adsorption and desorption processes on the PS surface take place in a reversible and reproducible way. A respective change in impedance has been observed as function of frequency, depending on excitation molecule (Fig. 1).

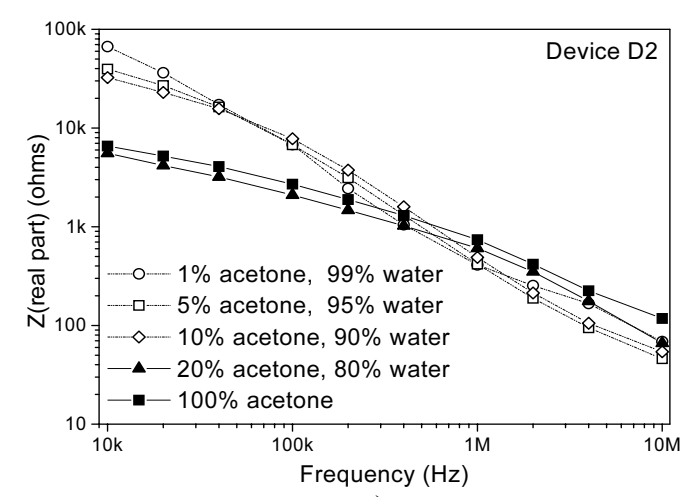

a)

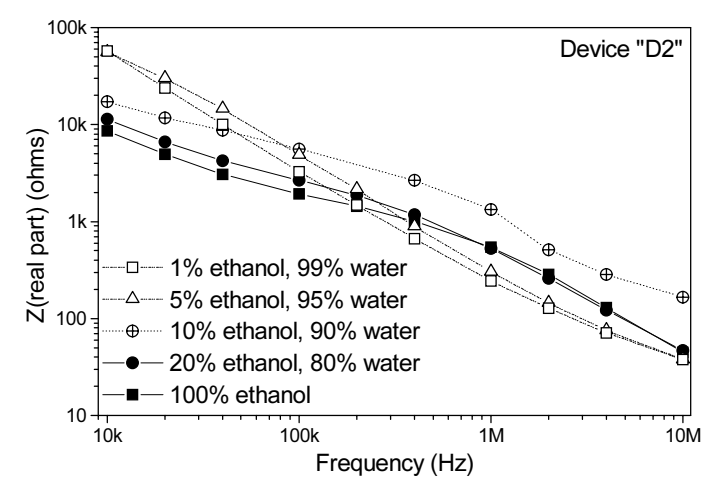

b)

Fig. 1 (a) Real part of impedance versus frequency of device D2 during tests with different acetone concentrations. (b) Real part of impedance versus frequency of device D2 during tests with different ethanol concentrations [9].

Based on this results, a model for impedance variation has been proposed and used to fit experimental data (Eq. (1)).

$$
Z(\omega) \alpha \omega^{s}
$$

where $\mathrm{Z}^{\prime}$ is the real part of the impedance and $\omega(=2 \pi \mathrm{f})$ is the measured angular frequency.

Modulation of the "s" parameter showed that identification of polar molecules is possible in the case of ethanol and acetone at room temperature. The law presented is considered an universal law which governs disordered materials such as PS [13]. The "s" exponential factor was calculated from experimental data in the range from $10 \mathrm{kHz}$ to $4 \mathrm{MHz}$ and ranged from -0.95 to -0.99 when devices were in vacuum. However, the value of this parameter varied, depending on type of molecules adsorbed in vapor environment and without dependence of geometry and gas concentration above $20 \%$. In environment saturated with acetone ( $20 \%$ to $100 \%$ in aqueous solution), "s" was $(-0.45 \pm 0.02)$ (see for example Fig. 2). Comparatively, the value of "s" remained approximately constant at $(-0.53 \pm 0.01)$ to $(-0.56 \pm 0.01)$ for essays in atmosphere saturated with ethanol (20\% to $100 \%$ in aqueous solutions). The initial relationship between the electrical resistance and the frequency was re-established after desorption of gaseous substances in the devices, being the results very close to the measured conductivity obtained before each test. One can suppose that PS layers neither react chemically with the molecules, nor react with any residue of the electrolyte, which could be trapped in the pores.

A possible interpretation for these results is based on the microstructure of the PS: the ac electrical conduction of PS is associated with the hopping transport of charge carriers trough a high disordered network of PS, among localized sites, whose transition rate depends both on the spatial distance and on the energy difference between the two sites involved [12]. It is assumed that polar molecules adsorbed by PS, might lower energy barriers between sites or particles, thus enhancing conductivity. 
The proposed model for analyzing impedance variation with frequency accounts for the experimental modulation observed, and supports the hypothesis of gas selectivity for sensors devices fabricated with PS as base material.

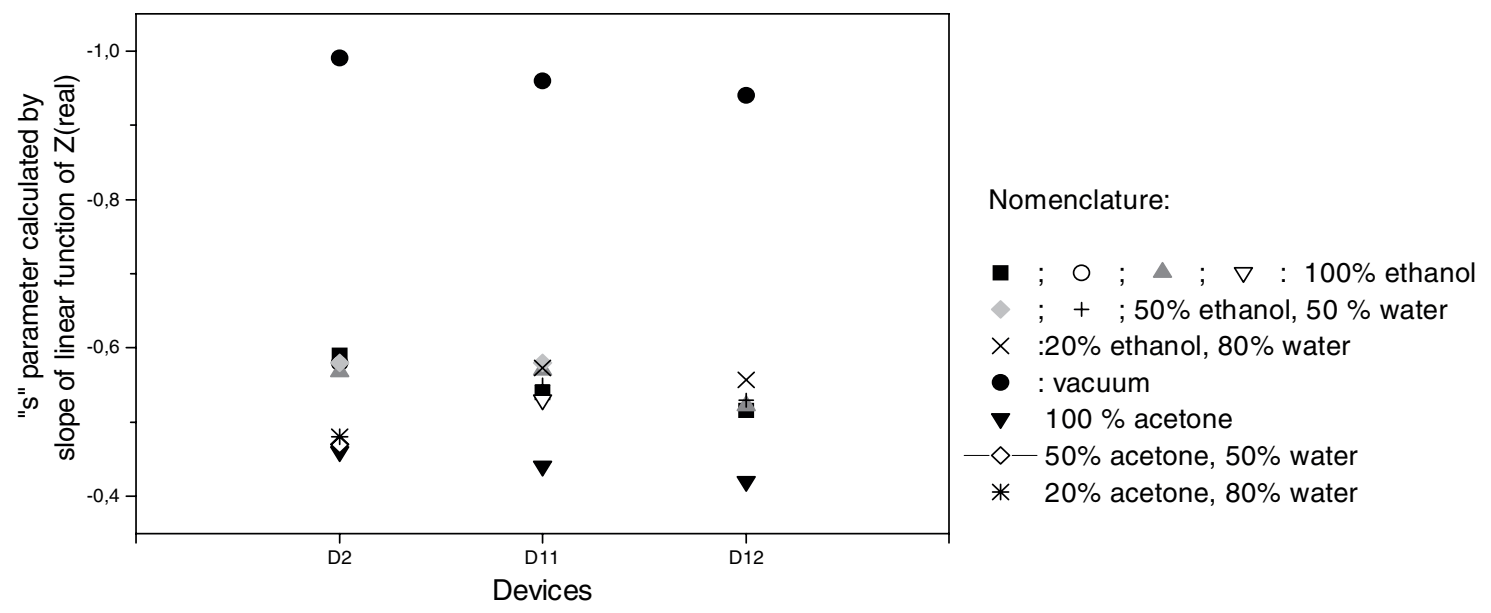

Fig. 2 Comparison of parameter "s" for devices with different area/perimeter ratio (named D2, D11 and D12). For each device, "s" is evaluated based on experimental results. Modulation of "s" is obtained through the effect of polar molecules, such as ethanol $(\mathrm{s}=-0.55)$ and acetone $(\mathrm{s}=-0.45)$, as opposed to the behaviour in vacuum $(\mathrm{s}=-0.9)$ [9].

\subsubsection{PL quenching effect in PS for optical gas sensor}

It is worth mentioning that optical and electrical properties of PS change dramatically in relation to Si bulk material due to quantum confinement and surface effect. In this sense, it is important to control the nanocrystal size and surface structure within PS films in order to apply it in optical gas sensor devices fabrication.

Based on results from Raman and optical absorption spectra, nanoporous films present band gap energy between valence band and conduction band of c.a $2.5 \mathrm{eV}$ with indirect characteristic. Experimental results of photoluminescence (PL) emission of PS films show a peak at c.a $1.7 \mathrm{eV}$ and its highest intensity is correlated with excitation laser source near to peak of absorption band $(2.4 \mathrm{eV})$. Moreover, the polarization coefficient (LPD: Linear Polarization Degree) of the PL emission shows a dependence on the laser excitation. The LPD values are higher for lower laser energies [14]. All the results pointed that PL emission in PS can be due to a radiative recombination considering the mechanism as phonon assisted process known as Jahn-Teller effect [15]. Therefore, the surface crystallite structure should control the PL emission.

In this work, in order to apply PS films to optical sensing and considering the mechanism of PL emission above mentioned, nanoporous films were exposed to different gas ambient and PL quenching effect was analyzed.

Figure 3a shows the efficiency of PL quenching as a function of quencher concentration (gas molecules). The PL quenching efficiency was measured by the $\mathrm{I}_{0} / \mathrm{I}$ ratio, where $\mathrm{I}_{0}$ and I represent the integrated intensities of the PL band before and after gas injection in the vacuum chamber, respectively. 


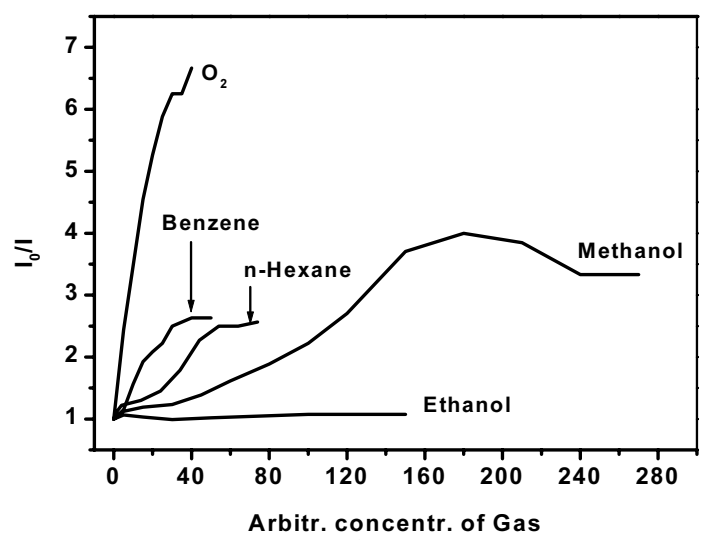

a)

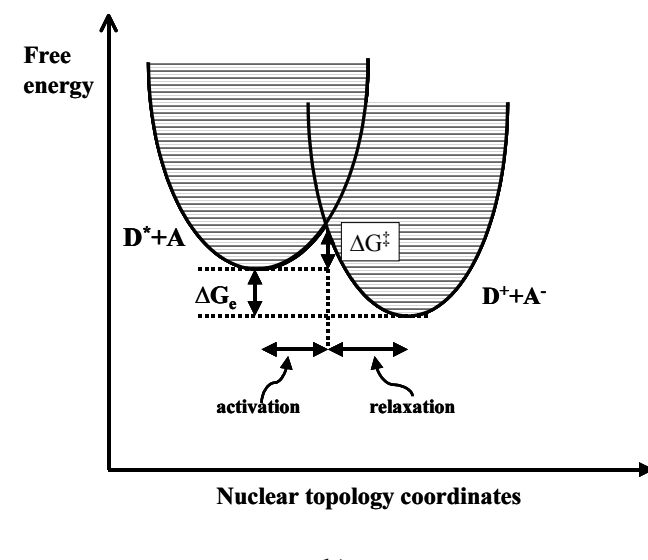

b)

Fig. 3 a) The efficiency of PL quenching as function of gas concentration. b) Free-energy surface profile for the electron transfer (ET) reaction. $\mathrm{D}^{*}$ and $\mathrm{A}$ are the donor and acceptor species before reaction; $\mathrm{D}^{+}$and $\mathrm{A}^{-}$represent the oxidized donor and reduced acceptor after reaction. $\Delta \mathrm{G}_{\mathrm{e}}$ is the barrier energy [17].

The efficiency of PL quenching presents two regions: the first region is a linear region that can be approximated by Stern-Volmer equation [16] and the second region is related to quenching saturation. The high polar molecules (ethanol and methanol) present a small slope in the Stern-Volmer sense when compared to non-polar molecules. On the other hand, it can be observed that the quenching-efficiency curves with larger slopes $\left(\mathrm{O}_{2}\right.$, Benzene and n-Hexane curves) reach the saturation region at low quencher concentrations.

From the optical absorption and dipole moment features of gas molecules used, it should be rule out the energy transfer and electrostatics process as responsible for the PL quenching effect. The electron transfer (ET) mechanism for PL quenching is governed by the Rehm-Weller equation, expressed as follows [16]:

$$
\Delta G_{e}=E^{0}\left(D^{*} / D^{+}\right)-E^{0}\left(A / A^{-}\right)-w_{p}
$$

where $\Delta G_{e}$ is the free energy changes involved in the electron transfer process; $E^{0}\left(D^{*} / D^{+}\right)$and $E^{0}\left(A / A^{-}\right)$ are redox potentials of the excited donor and acceptor, respectively; $\mathrm{w}_{\mathrm{p}}$ is the electrostatic work due to internal and external sphere reorganization.

In the present work, the $\mathrm{w}_{\mathrm{p}}$ contribution can be neglected since we are dealing with molecules in the gas phase and not in solution. From the redox potential of gas molecules and PS, the negative values of $\Delta \mathrm{G}_{\mathrm{e}}$ were obtained [17] showing that the ET process from excited PS to gas molecules is exothermic, i.e., the electron transfer from photo-exited PS film to the gas molecules is thermodynamically more favorable.

The quenching of PL emission of PS films exposed to gas molecules investigated in this work is explained by an electron transfer mechanism as follows: photo-excited PS film promotes electron-hole pairs localized at the $\mathrm{Si}-\mathrm{O}-\mathrm{SiR}$ moieties on the surface of crystallites. These moieties act as electron donors (D) and the gas molecules act as acceptors (A). When the gas molecules achieve the PS surface, their molecular orbitals (acceptor states) mix to donor excited states localized at the Si-O-SiR moieties promoting the electron transfer reaction and consequently the PL quenching (Fig. 3b). The fact that ethanol does not quench the PL emission of PS films can be explained by an unfavourable condition for ethanol alignment, avoiding the donor-acceptor states mixing.

The PL quenching effect showed a good selective and sensitivity behavior that promises the PS films as excellent material for optical gas sensor application. 


\subsection{Tin oxide gas sensors}

Gas sensors based on tin oxide $\left(\mathrm{SnO}_{2}\right)$ material are widely used and studied, mainly due to its high sensitivity for humidity and inflammable gases.

The $\mathrm{SnO}_{2}$ thin film is an intrinsically polycrystalline $\mathrm{n}$ type semiconductor with grain sizes ranging from nano to micrometer scale, depending on the synthesization parameters of the material. The mechanism of gas detection can be explained as two steps process: first the oxygen molecules from the dry fresh air are adsorbed on the grains surfaces, these adsorbed oxygen captures free electrons from conduction band of $\mathrm{SnO}_{2}$ reducing its electrical conductivity. Second, when reductive gas species are present, there is a "competition" for oxygen and, consequently, the $\mathrm{SnO}_{2}$ conductivity can be increased [18]. The variation of conductivity is strongly dependent of the gas specie and its concentration, as well as parameters like sensor temperature and fabrication conditions.

Although $\mathrm{SnO}_{2}$ sensors show high sensitivity response for inflammable gases, its electrical characteristics can vary considerably after some time of operation, i.e. the "drift" or "aging" effect of the sensor. For this reason, the practical usage of the sensor depends on the electrical conductivity normalization and adequate signal analysis. A common gas sensing system (or "electronic nose") can apply an array of sensors with different sensitivities for interest gases and the output signals can be interpreted by the artificial-neural-network (ANN) approach [19].

In order to improve sensors selectivity for different gas species, the addition of dopants into $\mathrm{SnO}_{2}$ was suggested [20]. In our group, gas sensors based on $\mathrm{SnO}_{2}$ film doped with nickel were studied and applied to $\mathrm{SO}_{2}$ detection. The $\mathrm{SnO}_{2}$ film doped with $1 \%$ of Ni (molar concentration) was prepared by Pechini's method [21] in such way that a liquid solution of $\mathrm{SnO}_{2}-\mathrm{Ni}$ and organic solvelts was obtained. This solution was deposited by spin coating technique over two interdigited gold contacts on $\mathrm{AlO}_{3}$ substrates (Fig. 4a). The polycrystalline $\mathrm{SnO}_{2}-\mathrm{Ni}$ films with nanometric grain sizes were obtained after device calcinations at $500{ }^{\circ} \mathrm{C}$ during 4 hours in dry air so the final devices were thin film resistors with gold electrodes. The electrical resistances of sensors were analyzed as a function of $\mathrm{SO}_{2}$ concentrations within experimental chamber with dry air and at ambient temperature. The range of $\mathrm{SO}_{2}$ concentrations varies from 0 to $164 \mathrm{ppm}$.

Figure $4 \mathrm{~b}$ shows the relative variation of the electrical resistance $\left(-\Delta \mathrm{R} / \mathrm{R}_{0}\right)$ versus $\mathrm{SO}_{2}$ concentration . It can be observed a linear response in the range of 0 to $30 \mathrm{ppm}$ of gas with a slope about $0.0058 \mathrm{ppm}^{-1}$. For values above of $30 \mathrm{ppm}$ the response showed a saturation of the sensor.

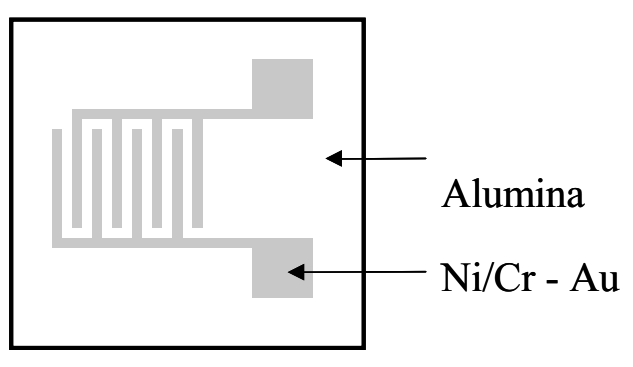

a)

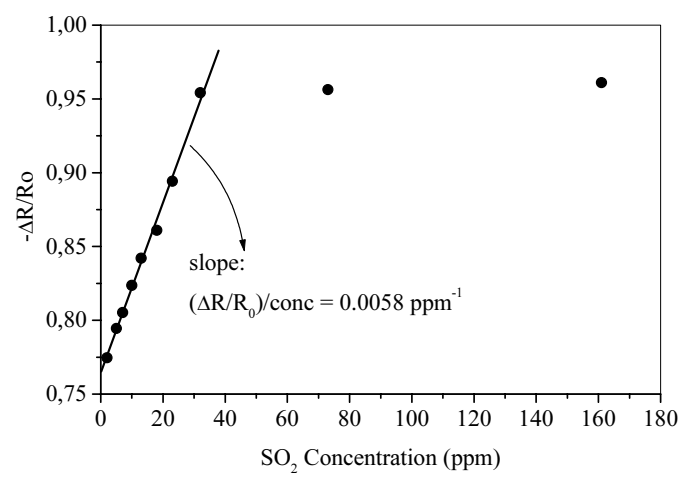

b)

Fig. 4 a) Sensor layout: interdigited gold electrodes. b) Relative electrical resistance variation of device vs. $\mathrm{SO}_{2}$ concentration. 
Thus, $\mathrm{Ni}-\mathrm{SnO}_{2}$ nanostructured films showed satisfactory sensitivity for $\mathrm{SO}_{2}$ detection, even operated at room temperature, what is a good characteristic for integrated gas sensors. As continuity, improvements on sensors selectivity can be achieved by doping $\mathrm{SnO}_{2}$ with other metals in order to obtain a complete gas sensor system.

\section{Conclusions}

Some integrated sensors as MEA and gas sensors based on nanomaterials were discussed.

With primary neuronal cultures developed over MEA it is possible to maintain cells in a very well controlled environment, without the need for glial cells or high-resolution microscopy. We moreover show that the charge injection limit can be lowered to less than $100 \mathrm{nC} / \mathrm{cm}^{2}$, which is helpful for determining thresholds for in vivo electrical stimuli in several neural prosthetic devices. Electrical stimulation of identified cells was also shown and two stimulus-driven responses were presented: inhibitory stimuli and beating behavior.

The sensing mechanism of PS can be either based on optical properties or on conductivity variations, as analysed in this paper. A model for impedance variation has been proposed and used to fit experimental data. Modulation of the "s" parameter showed that identification of polar molecules is possible in the case of ethanol and acetone, for example.

Based on PL of PS for gas sensing application, we propose the electron transfer mechanism as responsible for the PL quenching as function of gas species and concentration. The PL quenching phenomenon led itself to interesting optical sensor applications.

Acknowledgements The work was supported in part by CNPq and by FAPESP.

\section{References}

[1] T. Sauter and H. Nachtnebel, IEEE Trans. on Instrum. Meas. 52, 1854 (2003).

[2] M.P. Maher, H. Dvorak-Carbone, J. Pine, J.A. Wright, and Y.C. Tai, Med. Biol. Eng. Comp. 37, 110 (1999).

[3] N.I. Syed, A.G.M. Bulloch, and K. Lukowiak, Lymnaea Science 283, 282 (1990).

[4] N.L.V. Peixoto, S. Cordoba De Torresi, and F.J. Ramirez-Fernandez, in: Proceedings of BIOSIGNAL 2000, Brno, Czech Republic, June 21 to 23, 2000, pp. 86-89.

[5] A. Stett, W. Barth, S. Weiss, H. Haemmerle, and E. Zrenner, E. Vision Res. 40, 1785 (2000).

[6] O. Bisi, S. Ossicini, and L. Pavesi, Surf. Sci. Rep. 78, 1 (2000).

[7] M. Ben-Chorin, A. Kux, and I. Schechter, Appl. Phys. Lett. 64, 481 (1994).

[8] L. Boarino et al., Mater. Sci. Eng. B 69/70, 210 (2000).

[9] E. Galeazzo, H.E.M. Peres, N. Peixoto, and F.J. Ramirez-Fernandez, Sensors and Actuators B 93, 384 (2003).

[10] L. Pancheri et al., Sens. Actuators B 97, 45 (2004).

[11] R. Hérino, Mater. Sci. Eng. B 69/70, 70 (2000).

[12] M. Ben-Chorin, F. Moller, and F. Koch, Phys. Rev. B 51, 2199 (1995).

[13] N.F. Mott and E.A. Davis, Electronics Processes in Non-Crystalline Materials (2 ${ }^{\text {nd }}$ edition) (Clarendon Press, Oxford 1979).

[14] W. J. Salcedo, F. J. Ramirez-Fernadez, and J. C. Rubim, J. Raman Spectrosc. 32, 151 (2001).

[15] Y. E. Perlin and M. Wagner, The Dynamical Jahn-Teller Effect in Localized Systems; Modern Problems in Condensed Matter Sciences, Vol. 7 (Elsevier Science Publishers, B. V. 1984).

[16] G. J. Kavarnos; Fundamental of Photoinduced Electron Transfer (VCH Publishers, Weinheim 1993).

[17] W. J. Salcedo, F. J. Ramirez-Fernadez, and J. C. Rubim. Spectrochim. Acta, Part A 60, 1077 (2004).

[18] W. Gopel, T.A. Jones, and M. Kleitz, Chemical and Biochemical Sensors - Parts I and II, in: Sensors, Vol. 2/3, edited by W. Gopel, J. Hesse, and J.N. Zemel (VHC, Weinheim, 1991).

[19] G. Baratto and F.J. Ramirez-Fernandez, in: Proceedings of XIII SBMicro - International Conference on Microelectronics and Packaging - ICMP'98, 12-14 Agosto, Curitiba-PR, Brazil, 1998, pp. 102-109.

[20] I. Sayago et al., Sens. Actuators B 26/27, 19 (1995).

[21] M. Pechini, USpat. n`3330697, 1967. 